\title{
One-step implementation of the Fredkin gate via quantum Zeno dynamics
}

\author{
Zhi-Cheng Shi $^{1}$, Yan Xia ${ }^{1,2, *}$, and Jie Song ${ }^{3}$ \\ ${ }^{1}$ Department of Physics, Fuzhou University, Fuzhou 350002, China \\ ${ }^{2}$ School of Physics and Optoelectronic Technology, \\ Dalian University of Technology, Dalian 116024, China \\ ${ }^{3}$ Department of Physics, Harbin Institute of Technology, Harbin 150001, China
}

We study one-step implementation of the Fredkin gate in a bi-modal cavity under both resonant and large detuning conditions based on quantum Zeno dynamics, which reduces the complexity of experiment operations. The influence of cavity decay and atomic spontaneous emission is discussed by numerical calculation. The results demonstrate that the fidelity and the success probability are robust against cavity decay in both models and they are also insensitive to atomic spontaneous emission in the large detuning model. In addition, the interaction time is rather short in the resonant model compared to the large detuning model.

PACS numbers: 03. 65. Xp, 03. 67. Lx

Keywords: Quantum Zeno effect; Cavity quantum electrodynamics; Fredkin gate

\section{INTRODUCTION}

Recently, attentions have been mainly paid to physical implementation of quantum computer as it can provide a tremendous speedup when it is compared to classical computer [1], such as searching for data in an array. Until now, much significant progresses have been made in quantum computation during the last decade. It is well known that the cavity quantum electrodynamics (C-QED) which concerns the interaction of atoms and photons within cavities is the promising candidate for quantum information processing (QIP). There are numerous schemes proposed in the context of C-QED for achieving quantum computation, such as a scheme for realizing quantum logic gates and teleportation in C-QED [2], a

\footnotetext{
* corresponding author E-mail: xia-208@163.com
} 
scheme for realizing two-qubit quantum phase gate with a four-level system in C-QED [3], quantum logic gates for two atoms with a single resonant interaction [4], quantum phase gates for two atoms trapped in separate cavities connected by an optical fiber [5], etc..

For the case of three-qubit gates, much substantial efforts have been made in the study of the fundamental Toffoli [6] and Fredkin [7] gates. The quantum Toffoli gate performs a NOT operation on a target qubit depending on the states of two control qubits while the quantum Fredkin gate performs a SWAP operation on two target qubits depending on the state of a control qubit. A number of schemes [8-10] were proposed to implementation of a Toffoli gate and it was demonstrated in the experiment successfully [11]. The major progresses made in the Toffoli gate provide a attractive motivation for the attempts to implement the other three-qubit gate-Fredkin gate. So far, there are numerous schemes [8, 12, 13] proposed to implementation of Fredkin gate which is based on linear optical system. However, few schemes [14] are proposed to implementation of a Fredkin gate based on atomic systems. Atomic systems are suitable to act as qubits because moderate internal electronic states can coherently store information over very long time scale. At the same time, the switching on and off the atom-field interaction can be realizable through simple controlling classical laser field. The remarkable merits enlighten us to take a crack at constructing a theoretical model for implementing Fredkin gate in C-QED.

On the other hand, quantum Zeno effect (QZE) is an fascinating phenomenon which is applied to suppress decoherence via hindering transition between quantum states by performing frequent measurements [15]. It can be showed in two mainly aspects. On the one hand, the system will evolve away from its initial state and remains in the Zeno subspace determined by the measurement when frequently projected onto a multi-dimensional subspace [16, 17], which is called quantum Zeno dynamics. On the other hand, Facchi et al. [18] showed that QZE can also be reformulated in terms of a continuous coupling to obtain the same purpose without making use of von Neumann's projections and non-unitary dynamics in 2001. So far, numerous schemes have been proposed to implementation of quantum computation [19-24] via QZE.

In this paper, we study one-step implementation of a Fredkin gate in a bi-modal cavity under both resonant and large detuning conditions, which is based on quantum Zeno dynamics. The advantages in both models are threefold: (1) The gate can be implementation only one step without any single-qubit gate operation, which reduces the complexity of experi- 
ment operations. (2) The quantum information is encoded in the low states. Thus there is no energy relaxation for the atoms in the bi-modal cavity when the Fredkin gate operation is finished. (3) The state keeps in a subspace without exciting the cavity field during the whole system evolution, thus the fidelity and success probability are robust against cavity decay. On the other hand, in the large detuning model, the large detuning condition eliminates the excited state of atoms adiabatically, which is also insensitive to atomic spontaneous emission. The interaction time is rather short in the resonant model compared to the large detuning model.

This paper is organized as follows: In Sec. II, we study one-step implementation of the Fredkin gate in a bi-modal cavity under resonant conditions and large detuning based on quantum Zeno dynamics, respectively. In Sec. III, we analyze the influence of decoherence on the fidelity and success probability of the Fredkin gate by numerical calculation. A discuss on experimental feasibility and a summary are given in Sec. IV.

\section{IMPLEMENTATION OF A FREDKIN GATE IN A BI-MODAL CAVITY BASED ON QUANTUM ZENO DYNAMICS}

\section{A. Under the resonant condition}

As shown in Fig. 1, three identical atoms, which have an excited state $\left|e_{0}\right\rangle$, three ground states $\left|g_{L}\right\rangle,\left|g_{R}\right\rangle$ and $\left|g_{0}\right\rangle$, interact with a bi-modal cavity. The transitions $\left|e_{0}\right\rangle_{k} \leftrightarrow$ $\left|g_{L}\right\rangle_{k}\left(\left|g_{R}\right\rangle_{k}\right)(k=1,2,3)$ are resonantly coupled to left-circularly (right-circularly) polarized cavity modes. The transition $\left|e_{0}\right\rangle_{1} \leftrightarrow\left|g_{0}\right\rangle_{1}$ is resonantly driven by classical laser field with Rabi frequency $\Omega$. In the interaction picture, the Hamiltonian for the whole system can be written as $(\hbar=1)$

$$
\begin{aligned}
H_{\text {total }} & =H_{\text {laser }}+H_{c}, \\
H_{\text {laser }} & =\Omega\left(\left|e_{0}\right\rangle_{1}\left\langle g_{0}|+| g_{0}\right\rangle_{1}\left\langle e_{0}\right|\right), \\
H_{c} & =\sum_{k=1}^{3}\left(g_{k, L} a_{L}\left|e_{0}\right\rangle_{k}\left\langle g_{L}\left|+g_{k, R} a_{R}\right| e_{0}\right\rangle_{k}\left\langle g_{R}\right|\right)+\text { H.c. }
\end{aligned}
$$

$a_{L}^{\dagger}\left(a_{R}^{\dagger}\right)$ and $a_{L}\left(a_{R}\right)$ are the creation and annihilation operators for the bi-modal cavity mode. $g_{k, L}\left(g_{k, R}\right)$ is the coupling strength between the $k$ th atom and the left-(right-)circularly polarized cavity mode. We assumed $g_{k, j}=g \in R$ for simplicity. The quantum information 
is encoded in a subspace spanned by the states $\left\{\left|g_{R}\right\rangle_{1},\left|g_{0}\right\rangle_{1},\left|g_{L}\right\rangle_{2},\left|g_{R}\right\rangle_{2},\left|g_{L}\right\rangle_{3},\left|g_{R}\right\rangle_{3}\right\}$ while the cavity is in vacuum state $|00\rangle$.

For the initial states including $\left|g_{R}\right\rangle_{1}\left|g_{L}\right\rangle_{2}\left|g_{L}\right\rangle_{3}|00\rangle, \quad\left|g_{R}\right\rangle_{1}\left|g_{L}\right\rangle_{2}\left|g_{R}\right\rangle_{3}|00\rangle$, $\left|g_{R}\right\rangle_{1}\left|g_{R}\right\rangle_{2}\left|g_{L}\right\rangle_{3}|00\rangle$, and $\left|g_{R}\right\rangle_{1}\left|g_{R}\right\rangle_{2}\left|g_{R}\right\rangle_{3}|00\rangle$, they remain unchanged during the time evolution because $H_{\text {total }}\left|g_{R}\right\rangle_{1}\left|g_{L}\right\rangle_{2}\left|g_{L}\right\rangle_{3}|00\rangle=0, \quad H_{\text {total }}\left|g_{R}\right\rangle_{1}\left|g_{L}\right\rangle_{2}\left|g_{R}\right\rangle_{3}|00\rangle=0$, $H_{\text {total }}\left|g_{R}\right\rangle_{1}\left|g_{R}\right\rangle_{2}\left|g_{L}\right\rangle_{3}|00\rangle=0$, and $H_{\text {total }}\left|g_{R}\right\rangle_{1}\left|g_{R}\right\rangle_{2}\left|g_{R}\right\rangle_{3}|00\rangle=0$.

If the initial state is $\left|g_{0}\right\rangle_{1}\left|g_{L}\right\rangle_{2}\left|g_{R}\right\rangle_{3}|00\rangle$, it will evolve in a closed subspace spanned by

$$
\begin{aligned}
\left|\phi_{1}\right\rangle & =\left|g_{0}\right\rangle_{1}\left|g_{L}\right\rangle_{2}\left|g_{R}\right\rangle_{3}|00\rangle, \\
\left|\phi_{2}\right\rangle & =\left|e_{0}\right\rangle_{1}\left|g_{L}\right\rangle_{2}\left|g_{R}\right\rangle_{3}|00\rangle, \\
\left|\phi_{3}\right\rangle & =\left|g_{L}\right\rangle_{1}\left|g_{L}\right\rangle_{2}\left|g_{R}\right\rangle_{3}|10\rangle, \\
\left|\phi_{4}\right\rangle & =\left|g_{L}\right\rangle_{1}\left|e_{0}\right\rangle_{2}\left|g_{R}\right\rangle_{3}|00\rangle, \\
\left|\phi_{5}\right\rangle & =\left|g_{L}\right\rangle_{1}\left|g_{R}\right\rangle_{2}\left|g_{R}\right\rangle_{3}|01\rangle, \\
\left|\phi_{6}\right\rangle & =\left|g_{L}\right\rangle_{1}\left|g_{R}\right\rangle_{2}\left|e_{0}\right\rangle_{3}|00\rangle, \\
\left|\phi_{7}\right\rangle & =\left|g_{L}\right\rangle_{1}\left|g_{R}\right\rangle_{2}\left|g_{L}\right\rangle_{3}|10\rangle, \\
\left|\phi_{8}\right\rangle & =\left|g_{R}\right\rangle_{1}\left|g_{L}\right\rangle_{2}\left|g_{R}\right\rangle_{3}|01\rangle, \\
\left|\phi_{9}\right\rangle & =\left|g_{R}\right\rangle_{1}\left|g_{L}\right\rangle_{2}\left|e_{0}\right\rangle_{3}|00\rangle, \\
\left|\phi_{10}\right\rangle & =\left|g_{R}\right\rangle_{1}\left|g_{L}\right\rangle_{2}\left|g_{L}\right\rangle_{3}|10\rangle, \\
\left|\phi_{11}\right\rangle & =\left|g_{R}\right\rangle_{1}\left|e_{0}\right\rangle_{2}\left|g_{L}\right\rangle_{3}|00\rangle, \\
\left|\phi_{12}\right\rangle & =\left|g_{R}\right\rangle_{1}\left|g_{R}\right\rangle_{2}\left|g_{L}\right\rangle_{3}|01\rangle, \\
\left|\phi_{13}\right\rangle & =\left|e_{0}\right\rangle_{1}\left|g_{R}\right\rangle_{2}\left|g_{L}\right\rangle_{3}|00\rangle, \\
\left|\phi_{14}\right\rangle & =\left|g_{0}\right\rangle_{1}\left|g_{R}\right\rangle_{2}\left|g_{L}\right\rangle_{3}|00\rangle,
\end{aligned}
$$

The subscripts 1, 2, and 3 represent the atom 1, atom 2, and atom 3, respectively. The state $|10\rangle(|01\rangle)$ denotes having one left (right)-circularly photon while the state $|00\rangle$ describes none photon in the cavity. On the condition $\Omega \ll g$, the Hilbert subspace is split into seven invariant Zeno subspaces [25, 26]

$$
\begin{array}{r}
H_{p_{0}}=\left\{\left|\phi_{1}\right\rangle,\left|\phi_{14}\right\rangle,\left|\varphi_{1}\right\rangle,\left|\varphi_{2}\right\rangle\right\}, H_{p_{1}}=\left\{\left|\varphi_{3}\right\rangle\right\}, H_{p_{2}}=\left\{\left|\varphi_{4}\right\rangle\right\}, H_{p_{3}}=\left\{\left|\varphi_{5}\right\rangle,\left|\varphi_{6}\right\rangle\right\} \\
H_{p_{4}}=\left\{\left|\varphi_{7}\right\rangle,\left|\varphi_{8}\right\rangle\right\}, H_{p_{5}}=\left\{\left|\varphi_{9}\right\rangle,\left|\varphi_{10}\right\rangle\right\}, H_{p_{6}}=\left\{\left|\varphi_{11}\right\rangle,\left|\varphi_{12}\right\rangle\right\}
\end{array}
$$


where

$$
\begin{aligned}
& \left|\varphi_{1}\right\rangle=\frac{1}{\sqrt{6}}\left(-\left|\phi_{3}\right\rangle+\left|\phi_{5}\right\rangle-\left|\phi_{7}\right\rangle+\left|\phi_{8}\right\rangle-\left|\phi_{10}\right\rangle+\left|\phi_{12}\right\rangle\right), \\
& \left|\varphi_{2}\right\rangle=\frac{1}{\sqrt{6}}\left(-\left|\phi_{2}\right\rangle+\left|\phi_{4}\right\rangle-\left|\phi_{6}\right\rangle+\left|\phi_{9}\right\rangle-\left|\phi_{11}\right\rangle+\left|\phi_{13}\right\rangle\right), \\
& \left|\varphi_{3}\right\rangle=\frac{1}{2 \sqrt{3}}\left(\left|\phi_{2}\right\rangle+\left|\phi_{3}\right\rangle+\left|\phi_{4}\right\rangle+\left|\phi_{5}\right\rangle+\left|\phi_{6}\right\rangle\right. \\
& \left.+\left|\phi_{7}\right\rangle+\left|\phi_{8}\right\rangle+\left|\phi_{9}\right\rangle+\left|\phi_{10}\right\rangle+\left|\phi_{11}\right\rangle+\left|\phi_{12}\right\rangle+\left|\phi_{13}\right\rangle\right), \\
& \left|\varphi_{4}\right\rangle=\frac{1}{2 \sqrt{3}}\left(\left|\phi_{2}\right\rangle-\left|\phi_{3}\right\rangle+\left|\phi_{4}\right\rangle-\left|\phi_{5}\right\rangle+\left|\phi_{6}\right\rangle\right. \\
& \left.-\left|\phi_{7}\right\rangle-\left|\phi_{8}\right\rangle+\left|\phi_{9}\right\rangle-\left|\phi_{10}\right\rangle+\left|\phi_{11}\right\rangle-\left|\phi_{12}\right\rangle+\left|\phi_{13}\right\rangle\right), \\
& \left|\varphi_{5}\right\rangle=\frac{1}{2 \sqrt{2}}\left(\left|\phi_{2}\right\rangle-\left|\phi_{3}\right\rangle+\left|\phi_{5}\right\rangle-\left|\phi_{6}\right\rangle-\left|\phi_{9}\right\rangle+\left|\phi_{10}\right\rangle-\left|\phi_{12}\right\rangle+\left|\phi_{13}\right\rangle\right) \text {, } \\
& \left|\varphi_{6}\right\rangle=\frac{1}{2 \sqrt{6}}\left(\left|\phi_{2}\right\rangle+\left|\phi_{3}\right\rangle-2\left|\phi_{4}\right\rangle+\left|\phi_{5}\right\rangle+\left|\phi_{6}\right\rangle\right. \\
& \left.-2\left|\phi_{7}\right\rangle-2\left|\phi_{8}\right\rangle+\left|\phi_{9}\right\rangle+\left|\phi_{10}\right\rangle-2\left|\phi_{11}\right\rangle+\left|\phi_{12}\right\rangle+\left|\phi_{13}\right\rangle\right), \\
& \left|\varphi_{7}\right\rangle=\frac{1}{2 \sqrt{2}}\left(\left|\phi_{2}\right\rangle+\left|\phi_{3}\right\rangle-\left|\phi_{5}\right\rangle-\left|\phi_{6}\right\rangle-\left|\phi_{9}\right\rangle-\left|\phi_{10}\right\rangle+\left|\phi_{12}\right\rangle+\left|\phi_{13}\right\rangle\right), \\
& \left|\varphi_{8}\right\rangle=\frac{1}{2 \sqrt{6}}\left(\left|\phi_{2}\right\rangle-\left|\phi_{3}\right\rangle-2\left|\phi_{4}\right\rangle-\left|\phi_{5}\right\rangle+\left|\phi_{6}\right\rangle\right. \\
& \left.+2\left|\phi_{7}\right\rangle+2\left|\phi_{8}\right\rangle+\left|\phi_{9}\right\rangle-\left|\phi_{10}\right\rangle-2\left|\phi_{11}\right\rangle-\left|\phi_{12}\right\rangle+\left|\phi_{13}\right\rangle\right), \\
& \left|\varphi_{9}\right\rangle=\frac{1}{2 \sqrt{12-6 \sqrt{3}}}\left[-\left|\phi_{2}\right\rangle+\left|\phi_{3}\right\rangle+(1-\sqrt{3})\left|\phi_{4}\right\rangle-(\sqrt{3}-2)\left|\phi_{5}\right\rangle+(2-\sqrt{3})\left|\phi_{6}\right\rangle\right. \\
& -(\sqrt{3}-1)\left|\phi_{7}\right\rangle+(\sqrt{3}-1)\left|\phi_{8}\right\rangle-(2-\sqrt{3})\left|\phi_{9}\right\rangle+(\sqrt{3}-2)\left|\phi_{10}\right\rangle \\
& \left.-(1-\sqrt{3})\left|\phi_{11}\right\rangle-\left|\phi_{12}\right\rangle+\left|\phi_{13}\right\rangle\right] \\
& \left|\varphi_{10}\right\rangle=\frac{1}{2 \sqrt{12+6 \sqrt{3}}}\left[-\left|\phi_{2}\right\rangle-\left|\phi_{3}\right\rangle+(1+\sqrt{3})\left|\phi_{4}\right\rangle-(\sqrt{3}+2)\left|\phi_{5}\right\rangle+(2+\sqrt{3})\left|\phi_{6}\right\rangle\right. \\
& -(\sqrt{3}+1)\left|\phi_{7}\right\rangle+(\sqrt{3}+1)\left|\phi_{8}\right\rangle-(2+\sqrt{3})\left|\phi_{9}\right\rangle+(\sqrt{3}+2)\left|\phi_{10}\right\rangle \\
& \left.-(1+\sqrt{3})\left|\phi_{11}\right\rangle+\left|\phi_{12}\right\rangle+\left|\phi_{13}\right\rangle\right] \\
& \left|\varphi_{11}\right\rangle=\frac{1}{2 \sqrt{12-6 \sqrt{3}}}\left[-\left|\phi_{2}\right\rangle-\left|\phi_{3}\right\rangle+(1-\sqrt{3})\left|\phi_{4}\right\rangle+(\sqrt{3}-2)\left|\phi_{5}\right\rangle+(2-\sqrt{3})\left|\phi_{6}\right\rangle\right. \\
& +(\sqrt{3}-1)\left|\phi_{7}\right\rangle-(\sqrt{3}-1)\left|\phi_{8}\right\rangle-(2-\sqrt{3})\left|\phi_{9}\right\rangle-(\sqrt{3}-2)\left|\phi_{10}\right\rangle \\
& \left.-(1-\sqrt{3})\left|\phi_{11}\right\rangle+\left|\phi_{12}\right\rangle+\left|\phi_{13}\right\rangle\right] \\
& \left|\varphi_{12}\right\rangle=\frac{1}{2 \sqrt{12+6 \sqrt{3}}}\left[-\left|\phi_{2}\right\rangle+\left|\phi_{3}\right\rangle+(1+\sqrt{3})\left|\phi_{4}\right\rangle+(\sqrt{3}+2)\left|\phi_{5}\right\rangle+(2+\sqrt{3})\left|\phi_{6}\right\rangle\right. \\
& +(\sqrt{3}+1)\left|\phi_{7}\right\rangle-(\sqrt{3}+1)\left|\phi_{8}\right\rangle-(2+\sqrt{3})\left|\phi_{9}\right\rangle-(\sqrt{3}+2)\left|\phi_{10}\right\rangle \\
& \left.-(1+\sqrt{3})\left|\phi_{11}\right\rangle-\left|\phi_{12}\right\rangle+\left|\phi_{13}\right\rangle\right],
\end{aligned}
$$

corresponding to eigenvalues $\eta_{0}=0, \eta_{1}=2 g, \eta_{2}=-2 g, \eta_{3}=-g, \eta_{4}=g, \eta_{5}=-\sqrt{3} g$ and 
$\eta_{6}=\sqrt{3} g$ with the projections

$$
P_{n}=\sum_{j}\left|\beta_{i, j}\right\rangle\left\langle\beta_{i, j}\right|,\left(\left|\beta_{i, j}\right\rangle \in H_{P_{n}}\right)
$$

Therefore the Hamiltonian of the current system is approximately dominated by

$$
\begin{aligned}
H_{\text {total }} \cong & \sum_{n}\left(\eta_{n} P_{n}+P_{n} H_{\text {laser }} P_{n}\right) \\
= & 2 g\left|\varphi_{3}\right\rangle\left\langle\varphi_{3}|-2 g| \varphi_{4}\right\rangle\left\langle\varphi_{4}|-g| \varphi_{5}\right\rangle\left\langle\varphi_{5}|-g| \varphi_{6}\right\rangle\left\langle\varphi_{6}|+g| \varphi_{7}\right\rangle\left\langle\varphi_{7}\right| \\
& +g\left|\varphi_{8}\right\rangle\left\langle\varphi_{8}|-\sqrt{3} g| \varphi_{9}\right\rangle\left\langle\varphi_{9}|-\sqrt{3} g| \varphi_{10}\right\rangle\left\langle\varphi_{10}|+\sqrt{3} g| \varphi_{11}\right\rangle\left\langle\varphi_{11}|+\sqrt{3} g| \varphi_{12}\right\rangle\left\langle\varphi_{12}\right| \\
& +\frac{1}{\sqrt{6}}\left(-\Omega\left|\phi_{1}\right\rangle\left\langle\varphi_{2}|+\Omega| \phi_{14}\right\rangle\left\langle\varphi_{2}\right|+\text { H.c. }\right) .
\end{aligned}
$$

It is easily to find the Zeno subspace $H_{p_{0}}=\left\{\left|\phi_{1}\right\rangle,\left|\phi_{14}\right\rangle,\left|\varphi_{1}\right\rangle,\left|\varphi_{2}\right\rangle\right\}$ with eigenvalues $\eta_{0}=0$. Thus the system state will evolve in that Zeno subspace with $H_{\text {eff }}$ :

$$
H_{e f f}=\frac{1}{\sqrt{6}}\left(-\Omega\left|\phi_{1}\right\rangle\left\langle\varphi_{2}|+\Omega| \phi_{14}\right\rangle\left\langle\varphi_{2}\right|+\text { H.c. }\right) .
$$

For an interaction time $t$, the state of the whole system becomes

$$
|\Phi(t)\rangle=\left(\frac{1}{2}+\frac{1}{2} \cos \frac{\sqrt{3}}{3} \Omega t\right)\left|\phi_{1}\right\rangle-\frac{1}{2}\left(\cos \frac{\sqrt{3}}{3} \Omega t-1\right)\left|\phi_{14}\right\rangle+i \frac{\sqrt{2}}{2} \sin \frac{\sqrt{3}}{3} \Omega t\left|\varphi_{2}\right\rangle .
$$

If we choose $\frac{\sqrt{3}}{3} \Omega t=\pi$ and the final state becomes $\left|\Phi\left(\frac{\sqrt{3} \pi}{\Omega}\right)\right\rangle=\left|\phi_{14}\right\rangle$, one will obtain the transform: $\left|\phi_{1}\right\rangle=\left|g_{0}\right\rangle_{1}\left|g_{L}\right\rangle_{2}\left|g_{R}\right\rangle_{3}|00\rangle \rightarrow\left|\phi_{14}\right\rangle=\left|g_{0}\right\rangle_{1}\left|g_{R}\right\rangle_{2}\left|g_{L}\right\rangle_{3}|00\rangle$.

If the initial state is $\left|g_{0}\right\rangle_{1}\left|g_{R}\right\rangle_{2}\left|g_{L}\right\rangle_{3}|00\rangle$, a analogue method is utilized with the initial state $\left|g_{0}\right\rangle_{1}\left|g_{L}\right\rangle_{2}\left|g_{R}\right\rangle_{3}|00\rangle$. As a result, the interaction time is also $\frac{\sqrt{3} \pi}{\Omega}$ when the final state becomes $\left|g_{0}\right\rangle_{1}\left|g_{L}\right\rangle_{2}\left|g_{R}\right\rangle_{3}|00\rangle$.

If the initial state is $\left|g_{0}\right\rangle_{1}\left|g_{L}\right\rangle_{2}\left|g_{L}\right\rangle_{3}|00\rangle$, it will evolve in a closed subspace spanned by

$$
\begin{aligned}
\left|\phi_{1}^{\prime}\right\rangle & =\left|g_{0}\right\rangle_{1}\left|g_{L}\right\rangle_{2}\left|g_{L}\right\rangle_{3}|00\rangle, \\
\left|\phi_{2}^{\prime}\right\rangle & =\left|e_{0}\right\rangle_{1}\left|g_{L}\right\rangle_{2}\left|g_{L}\right\rangle_{3}|00\rangle, \\
\left|\phi_{3}^{\prime}\right\rangle & =\left|g_{L}\right\rangle_{1}\left|g_{L}\right\rangle_{2}\left|g_{L}\right\rangle_{3}|10\rangle, \\
\left|\phi_{4}^{\prime}\right\rangle & =\left|g_{L}\right\rangle_{1}\left|e_{0}\right\rangle_{2}\left|g_{L}\right\rangle_{3}|00\rangle, \\
\left|\phi_{5}^{\prime}\right\rangle & =\left|g_{L}\right\rangle_{1}\left|g_{R}\right\rangle_{2}\left|g_{L}\right\rangle_{3}|01\rangle, \\
\left|\phi_{6}^{\prime}\right\rangle & =\left|g_{L}\right\rangle_{1}\left|g_{L}\right\rangle_{2}\left|e_{0}\right\rangle_{3}|00\rangle, \\
\left|\phi_{7}^{\prime}\right\rangle & =\left|g_{L}\right\rangle_{1}\left|g_{L}\right\rangle_{2}\left|g_{R}\right\rangle_{3}|01\rangle, \\
\left|\phi_{8}^{\prime}\right\rangle & =\left|g_{R}\right\rangle_{1}\left|g_{L}\right\rangle_{2}\left|g_{L}\right\rangle_{3}|01\rangle .
\end{aligned}
$$


On the condition $\Omega \ll g$, the Hilbert subspace is split into five invariant Zeno subspaces [25, 26]

$$
H_{p_{0}}^{\prime}=\left\{\left|\phi_{1}^{\prime}\right\rangle,\left|\varphi_{1}^{\prime}\right\rangle\right\}, H_{p_{1}}^{\prime}=\left\{\left|\varphi_{2}^{\prime}\right\rangle,\left|\varphi_{3}^{\prime}\right\rangle\right\}, H_{p_{3}}^{\prime}=\left\{\left|\varphi_{4}^{\prime}\right\rangle,\left|\varphi_{5}^{\prime}\right\rangle\right\}, H_{p_{4}}^{\prime}=\left\{\left|\varphi_{6}^{\prime}\right\rangle\right\}, H_{p_{5}}^{\prime}=\left\{\left|\varphi_{7}^{\prime}\right\rangle\right\}
$$

where

$$
\begin{aligned}
&\left|\varphi_{1}^{\prime}\right\rangle=\frac{1}{2}\left(-\left|\phi_{3}^{\prime}\right\rangle+\left|\phi_{5}^{\prime}\right\rangle+\left|\phi_{7}^{\prime}\right\rangle+\left|\phi_{8}^{\prime}\right\rangle\right), \\
&\left|\varphi_{2}^{\prime}\right\rangle=\frac{1}{2 \sqrt{3}}\left(-\left|\phi_{2}^{\prime}\right\rangle+2\left|\phi_{4}^{\prime}\right\rangle-2\left|\phi_{5}^{\prime}\right\rangle-\left|\phi_{6}^{\prime}\right\rangle+\left|\phi_{7}^{\prime}\right\rangle+\left|\phi_{8}^{\prime}\right\rangle\right), \\
&\left|\varphi_{3}^{\prime}\right\rangle=\frac{1}{2}\left(-\left|\phi_{2}^{\prime}\right\rangle+\left|\phi_{6}^{\prime}\right\rangle-\left|\phi_{7}^{\prime}\right\rangle+\left|\phi_{8}^{\prime}\right\rangle\right), \\
&\left|\varphi_{4}^{\prime}\right\rangle\left.=\frac{1}{2 \sqrt{3}}\left(\left|\phi_{2}^{\prime}\right\rangle-2\left|\phi_{4}^{\prime}\right\rangle-2 \phi_{5}^{\prime}\right\rangle+\left|\phi_{6}^{\prime}\right\rangle+\left|\phi_{7}^{\prime}\right\rangle+\left|\phi_{8}^{\prime}\right\rangle\right), \\
&\left|\varphi_{5}^{\prime}\right\rangle=\frac{1}{2}\left(\left|\phi_{2}^{\prime}\right\rangle-\left|\phi_{6}^{\prime}\right\rangle-\left|\phi_{7}^{\prime}\right\rangle+\left|\phi_{8}^{\prime}\right\rangle\right), \\
&\left|\varphi_{6}^{\prime}\right\rangle=\frac{1}{2 \sqrt{6}}\left(-\left|\phi_{2}^{\prime}\right\rangle+3\left|\phi_{3}^{\prime}\right\rangle-2\left|\phi_{4}^{\prime}\right\rangle+\left|\phi_{5}^{\prime}\right\rangle-2\left|\phi_{6}^{\prime}\right\rangle+\left|\phi_{7}^{\prime}\right\rangle+\left|\phi_{8}^{\prime}\right\rangle\right), \\
&\left|\varphi_{7}^{\prime}\right\rangle=\frac{1}{2 \sqrt{6}}\left(\left|\phi_{2}^{\prime}\right\rangle+3\left|\phi_{3}^{\prime}\right\rangle+2\left|\phi_{4}^{\prime}\right\rangle+\left|\phi_{5}^{\prime}\right\rangle+2\left|\phi_{6}^{\prime}\right\rangle+\left|\phi_{7}^{\prime}\right\rangle+\left|\phi_{8}^{\prime}\right\rangle\right),
\end{aligned}
$$

corresponding to eigenvalues $\eta_{0}=0, \eta_{1}^{\prime}=-g, \eta_{2}^{\prime}=g, \eta_{3}^{\prime}=-2 g, \eta_{4}^{\prime}=2 g$, with the projections

$$
P_{n}^{\prime}=\sum_{j}\left|\beta_{i, j}^{\prime}\right\rangle\left\langle\beta_{i, j}^{\prime}\right|,\left(\left|\beta_{i, j}^{\prime}\right\rangle \in H_{P_{n}}^{\prime}\right) .
$$

Therefore the Hamiltonian of the current system is approximately dominated by

$$
\begin{aligned}
H_{\text {total }}^{\prime} & \cong \sum_{n}\left(\eta_{n}^{\prime} P_{n}^{\prime}+P_{n}^{\prime} H_{\text {laser }} P_{n}^{\prime}\right) \\
& =-g\left|\varphi_{2}^{\prime}\right\rangle\left\langle\varphi_{2}^{\prime}|-g| \varphi_{3}^{\prime}\right\rangle\left\langle\varphi_{3}^{\prime}|+g| \varphi_{4}^{\prime}\right\rangle\left\langle\varphi_{4}^{\prime}|+g| \varphi_{5}^{\prime}\right\rangle\left\langle\varphi_{5}^{\prime}|-2 g| \varphi_{6}^{\prime}\right\rangle\left\langle\varphi_{6}^{\prime}|+2 g| \varphi_{7}^{\prime}\right\rangle\left\langle\varphi_{7}^{\prime}\right| \cdot(13
\end{aligned}
$$

It is easily to find the Zeno subspace $H_{p_{0}}^{\prime}=\left\{\left|\phi_{1}^{\prime}\right\rangle,\left|\varphi_{1}^{\prime}\right\rangle\right\}$ with eigenvalues $\eta_{0}=0$. As $H_{\text {total } 1}^{\prime}\left|\phi_{1}^{\prime}\right\rangle=0$, the final state remains in the state $\left|g_{0}\right\rangle_{1}\left|g_{L}\right\rangle_{2}\left|g_{L}\right\rangle_{3}|00\rangle$ without any change during the evolution.

If the initial state is $\left|g_{0}\right\rangle_{1}\left|g_{R}\right\rangle_{2}\left|g_{R}\right\rangle_{3}|00\rangle$, a analogue method is utilized with the initial state $\left|g_{0}\right\rangle_{1}\left|g_{L}\right\rangle_{2}\left|g_{L}\right\rangle_{3}|00\rangle$. As a result, the final state also remains in the state $\left|g_{0}\right\rangle_{1}\left|g_{R}\right\rangle_{2}\left|g_{R}\right\rangle_{3}|00\rangle$ without any change. 
Thus the three qubits can be described as follows:

$$
\begin{aligned}
\left|g_{R}\right\rangle_{1}\left|g_{R}\right\rangle_{2}\left|g_{R}\right\rangle_{3}|00\rangle & \rightarrow\left|g_{R}\right\rangle_{1}\left|g_{R}\right\rangle_{2}\left|g_{R}\right\rangle_{3}|00\rangle, \\
\left|g_{R}\right\rangle_{1}\left|g_{R}\right\rangle_{2}\left|g_{L}\right\rangle_{3}|00\rangle & \rightarrow\left|g_{R}\right\rangle_{1}\left|g_{R}\right\rangle_{2}\left|g_{L}\right\rangle_{3}|00\rangle, \\
\left|g_{R}\right\rangle_{1}\left|g_{L}\right\rangle_{2}\left|g_{R}\right\rangle_{3}|00\rangle & \rightarrow\left|g_{R}\right\rangle_{1}\left|g_{L}\right\rangle_{2}\left|g_{R}\right\rangle_{3}|00\rangle, \\
\left|g_{R}\right\rangle_{1}\left|g_{L}\right\rangle_{2}\left|g_{L}\right\rangle_{3}|00\rangle & \rightarrow\left|g_{R}\right\rangle_{1}\left|g_{L}\right\rangle_{2}\left|g_{L}\right\rangle_{3}|00\rangle, \\
\left|g_{0}\right\rangle_{1}\left|g_{R}\right\rangle_{2}\left|g_{R}\right\rangle_{3}|00\rangle & \rightarrow\left|g_{0}\right\rangle_{1}\left|g_{R}\right\rangle_{2}\left|g_{R}\right\rangle_{3}|00\rangle, \\
\left|g_{0}\right\rangle_{1}\left|g_{R}\right\rangle_{2}\left|g_{L}\right\rangle_{3}|00\rangle & \rightarrow\left|g_{0}\right\rangle_{1}\left|g_{L}\right\rangle_{2}\left|g_{R}\right\rangle_{3}|00\rangle, \\
\left|g_{0}\right\rangle_{1}\left|g_{L}\right\rangle_{2}\left|g_{R}\right\rangle_{3}|00\rangle & \rightarrow\left|g_{0}\right\rangle_{1}\left|g_{R}\right\rangle_{2}\left|g_{L}\right\rangle_{3}|00\rangle, \\
\left|g_{0}\right\rangle_{1}\left|g_{L}\right\rangle_{2}\left|g_{L}\right\rangle_{3}|00\rangle & \rightarrow\left|g_{0}\right\rangle_{1}\left|g_{L}\right\rangle_{2}\left|g_{L}\right\rangle_{3}|00\rangle
\end{aligned}
$$

So we acquire the Fredkin gate from the Eq. (14) in the resonant model.

\section{B. Under the large detuning condition}

As shown in Fig. 2, the transitions $\left|e_{0}\right\rangle_{k} \leftrightarrow\left|g_{L}\right\rangle_{k}\left(\left|g_{R}\right\rangle_{k}\right)(k=1,2,3)$ are coupled to leftcircularly (right-circularly) polarized cavity modes. The transition $\left|e_{0}\right\rangle_{1} \leftrightarrow\left|g_{0}\right\rangle_{1}$ is driven by classical laser field with Rabi frequency $\Omega$. $\Delta$ denotes the detuning of the cavity modes

and classical laser field from the respective atomic transition. In the interaction picture, the Hamiltonian for the whole system can be written as $(\hbar=1)$

$$
\begin{aligned}
H_{t o t} & =H_{c a}+H_{l a}+H_{d e}, \\
H_{c a} & =\sum_{k=1}^{3}\left(g_{k, L} a_{L}\left|e_{0}\right\rangle_{k}\left\langle g_{L}\left|+g_{k, R} a_{R}\right| e_{0}\right\rangle_{k}\left\langle g_{R}\right|\right)+H . c, \\
H_{l a} & =\Omega\left(\left|e_{0}\right\rangle_{1}\left\langle g_{0}|+| g_{0}\right\rangle_{1}\left\langle e_{0}\right|\right), \\
H_{d e} & =\Delta\left(\left|e_{0}\right\rangle_{1}\left\langle e_{0}|+| e_{0}\right\rangle_{2}\left\langle e_{0}|+| e_{0}\right\rangle_{3}\left\langle e_{0}\right|\right) .
\end{aligned}
$$

The initial states including $\left|g_{R}\right\rangle_{1}\left|g_{R}\right\rangle_{2}\left|g_{R}\right\rangle_{3}|00\rangle,\left|g_{R}\right\rangle_{1}\left|g_{R}\right\rangle_{2}\left|g_{L}\right\rangle_{3}|00\rangle,\left|g_{R}\right\rangle_{1}\left|g_{L}\right\rangle_{2}\left|g_{R}\right\rangle_{3}|00\rangle$, and $\left|g_{R}\right\rangle_{1}\left|g_{L}\right\rangle_{2}\left|g_{L}\right\rangle_{3}|00\rangle$ remain unchanged during the time evolution because $H_{\text {tot }}\left|g_{R}\right\rangle_{1}\left|g_{R}\right\rangle_{2}\left|g_{R}\right\rangle_{3}|00\rangle=0, H_{t o t}\left|g_{R}\right\rangle_{1}\left|g_{R}\right\rangle_{2}\left|g_{L}\right\rangle_{3}|00\rangle=0, H_{t o t}\left|g_{R}\right\rangle_{1}\left|g_{L}\right\rangle_{2}\left|g_{R}\right\rangle_{3}|00\rangle=0$, and $H_{t o t}\left|g_{R}\right\rangle_{1}\left|g_{L}\right\rangle_{2}\left|g_{L}\right\rangle_{3}|00\rangle=0$.

If the initial state is $\left|g_{0}\right\rangle_{1}\left|g_{L}\right\rangle_{2}\left|g_{R}\right\rangle_{3}|00\rangle$, the whole system evolves in a closed subspace spanned by $\quad\left\{\left|\phi_{1}\right\rangle,\left|\phi_{2}\right\rangle,\left|\phi_{3}\right\rangle,\left|\phi_{4}\right\rangle,\left|\phi_{5}\right\rangle,\left|\phi_{6}\right\rangle,\left|\phi_{7}\right\rangle,\left|\phi_{8}\right\rangle,\left|\phi_{9}\right\rangle,\left|\phi_{10}\right\rangle,\left|\phi_{11}\right\rangle,\left|\phi_{12}\right\rangle,\left|\phi_{13}\right\rangle,\left|\phi_{14}\right\rangle\right\}$. 
Therefore we can rewrite the above Hamiltonian in the " $H_{c a}^{1}$ " representation:

$$
\begin{aligned}
H_{t o t}^{1}= & H_{c a}^{1}+H_{l a}^{1}+H_{d e}^{1}, \\
H_{c a}^{1}= & 2 g\left|\varphi_{3}\right\rangle\left\langle\varphi_{3}|-2 g| \varphi_{4}\right\rangle\left\langle\varphi_{4}|-g| \varphi_{5}\right\rangle\left\langle\varphi_{5}|-g| \varphi_{6}\right\rangle\left\langle\varphi_{6}|+g| \varphi_{7}\right\rangle\left\langle\varphi_{7}\right| \\
& +g\left|\varphi_{8}\right\rangle\left\langle\varphi_{8}|-\sqrt{3} g| \varphi_{9}\right\rangle\left\langle\varphi_{9}|-\sqrt{3} g| \varphi_{10}\right\rangle\left\langle\varphi_{10}|+\sqrt{3} g| \varphi_{11}\right\rangle\left\langle\varphi_{11}|+\sqrt{3} g| \varphi_{12}\right\rangle\left\langle\varphi_{12}\right|, \\
H_{l a}^{1}= & \frac{\Omega}{\sqrt{6}}\left|\varphi_{2}\right\rangle\left(\left\langle\phi_{14}\left|-\left\langle\phi_{1}\right|\right)+\frac{\Omega}{2 \sqrt{3}}\left(\left|\varphi_{3}\right\rangle+\left|\varphi_{4}\right\rangle\right)\left(\left\langle\phi_{14}\right|+\left\langle\phi_{1}\right|\right)\right.\right. \\
& +\frac{\Omega}{2 \sqrt{2}}\left(\left|\varphi_{5}\right\rangle+\left|\varphi_{7}\right\rangle\right)\left(\left\langle\phi_{14}\left|+\left\langle\phi_{1}\right|\right)+\frac{\Omega}{2 \sqrt{6}}\left(\left|\varphi_{6}\right\rangle+\left|\varphi_{8}\right\rangle\right)\left(\left\langle\phi_{14}\right|+\left\langle\phi_{1}\right|\right)\right.\right. \\
& +\frac{\Omega}{2 \sqrt{12-6 \sqrt{3}}}\left(\left|\varphi_{9}\right\rangle+\left|\varphi_{11}\right\rangle\right)\left(\left\langle\phi_{14}\right|-\left\langle\phi_{1}\right|\right) \\
& +\frac{\Omega}{2 \sqrt{12+6 \sqrt{3}}}\left(\left|\varphi_{10}\right\rangle+\left|\varphi_{12}\right\rangle\right)\left(\left\langle\phi_{14}\right|-\left\langle\phi_{1}\right|\right)+H . c ., \\
H_{d e}^{1}= & \Delta\left|\varphi_{2}\right\rangle\left\langle\varphi _ { 2 } \left|+\frac{\Delta}{2}\left(\left|\varphi_{3}\right\rangle+\left|\varphi_{4}\right\rangle\right)\left(\left\langle\varphi_{3}\left|+\left\langle\varphi_{4}\right|\right)+\frac{\Delta}{2}\left(\left|\varphi_{5}\right\rangle+\left|\varphi_{7}\right\rangle\right)\left(\left\langle\varphi_{5}\right|+\left\langle\varphi_{7}\right|\right)\right.\right.\right.\right. \\
& +\frac{\Delta}{2}\left(\left|\varphi_{6}\right\rangle+\left|\varphi_{8}\right\rangle\right)\left(\left\langle\varphi_{6}\left|+\left\langle\varphi_{8}\right|\right)+\frac{\Delta}{2}\left(\left|\varphi_{9}\right\rangle+\left|\varphi_{11}\right\rangle\right)\left(\left\langle\varphi_{9}\right|+\left\langle\varphi_{11}\right|\right)\right.\right. \\
& +\frac{\Delta}{2}\left(\left|\varphi_{10}\right\rangle+\left|\varphi_{12}\right\rangle\right)\left(\left\langle\varphi_{10}\right|+\left\langle\varphi_{12}\right|\right) .
\end{aligned}
$$

Furthermore, we assume that $U_{c a}^{1}=e^{-i H_{c a}^{1} t}$ is the unitary time evolution operator with respect to the Hamiltonian $H_{c a}^{1}$. After a calculation in the intermediate "picture", we obtain

$$
\begin{aligned}
H_{l a}^{1 I}= & U_{c a}^{1 \dagger} H_{l a}^{1} U_{c a}^{1} \\
= & \frac{\Omega}{\sqrt{6}}\left|\varphi_{2}\right\rangle\left(\left\langle\phi_{14}\left|-\left\langle\phi_{1}\right|\right)+\frac{\Omega}{2 \sqrt{3}}\left(e^{-i 2 g t}\left|\varphi_{3}\right\rangle+e^{i 2 g t}\left|\varphi_{4}\right\rangle\right)\left(\left\langle\phi_{14}\right|+\left\langle\phi_{1}\right|\right)\right.\right. \\
& +\frac{\Omega}{2 \sqrt{2}}\left(e^{i g t}\left|\varphi_{5}\right\rangle+e^{-i g t}\left|\varphi_{7}\right\rangle\right)\left(\left\langle\phi_{14}\left|+\left\langle\phi_{1}\right|\right)+\frac{\Omega}{2 \sqrt{6}}\left(e^{i g t}\left|\varphi_{6}\right\rangle+e^{-i g t}\left|\varphi_{8}\right\rangle\right)\left(\left\langle\phi_{14}\right|+\left\langle\phi_{1}\right|\right)\right.\right. \\
& +\frac{\Omega}{2 \sqrt{12-6 \sqrt{3}}}\left(e^{i \sqrt{3} g t}\left|\varphi_{9}\right\rangle+e^{-i \sqrt{3} g t}\left|\varphi_{11}\right\rangle\right)\left(\left\langle\phi_{14}\right|-\left\langle\phi_{1}\right|\right) \\
& +\frac{\Omega}{2 \sqrt{12+6 \sqrt{3}}}\left(e^{i \sqrt{3} g t}\left|\varphi_{10}\right\rangle+e^{-i \sqrt{3} g t}\left|\varphi_{12}\right\rangle\right)\left(\left\langle\phi_{14}\right|-\left\langle\phi_{1}\right|\right)+H . c ., \\
H_{d e}^{1 I}= & U_{c a}^{1 \dagger} H_{d e}^{1} U_{c a}^{1}
\end{aligned}
$$




$$
\begin{aligned}
= & \Delta\left|\varphi_{2}\right\rangle\left\langle\varphi_{2}\right|+\frac{\Delta}{2}\left(\left|\varphi_{3}\right\rangle\left\langle\varphi_{3}|+| \varphi_{4}\right\rangle\left\langle\varphi_{4}\right|\right)+\frac{\Delta}{2}\left(e^{-i 4 g t}\left|\varphi_{3}\right\rangle\left\langle\varphi_{4}\left|+e^{i 4 g t}\right| \varphi_{4}\right\rangle\left\langle\varphi_{3}\right|\right) \\
& +\frac{\Delta}{2}\left(\left|\varphi_{5}\right\rangle\left\langle\varphi_{5}|+| \varphi_{7}\right\rangle\left\langle\varphi_{7}\right|\right)+\frac{\Delta}{2}\left(e^{i 2 g t}\left|\varphi_{5}\right\rangle\left\langle\varphi_{7}\left|+e^{-i 2 g t}\right| \varphi_{7}\right\rangle\left\langle\varphi_{5}\right|\right) \\
& +\frac{\Delta}{2}\left(\left|\varphi_{6}\right\rangle\left\langle\varphi_{6}|+| \varphi_{8}\right\rangle\left\langle\varphi_{8}\right|\right)+\frac{\Delta}{2}\left(e^{i 2 g t}\left|\varphi_{6}\right\rangle\left\langle\varphi_{8}\left|+e^{-i 2 g t}\right| \varphi_{8}\right\rangle\left\langle\varphi_{6}\right|\right) \\
& +\frac{\Delta}{2}\left(\left|\varphi_{9}\right\rangle\left\langle\varphi_{9}|+| \varphi_{11}\right\rangle\left\langle\varphi_{11}\right|\right)+\frac{\Delta}{2}\left(e^{i 2 \sqrt{3} g t}\left|\varphi_{9}\right\rangle\left\langle\varphi_{11}\left|+e^{-i 2 \sqrt{3} g t}\right| \varphi_{11}\right\rangle\left\langle\varphi_{9}\right|\right) \\
& +\frac{\Delta}{2}\left(\left|\varphi_{10}\right\rangle\left\langle\varphi_{10}|+| \varphi_{12}\right\rangle\left\langle\varphi_{12}\right|\right)+\frac{\Delta}{2}\left(e^{i 2 \sqrt{3} g t}\left|\varphi_{10}\right\rangle\left\langle\varphi_{12}\left|+e^{-i 2 \sqrt{3} g t}\right| \varphi_{12}\right\rangle\left\langle\varphi_{10}\right|\right) .
\end{aligned}
$$

When the condition $\Omega \ll g$ is satisfied, we can safely discard the terms in $H_{l a}^{1 I}$ and $H_{d e}^{1 I}$ with high oscillating frequency $g, 2 g, 4 g, \sqrt{3} g$, and $2 \sqrt{3} g$. Then we move back to the original interaction picture from the intermediate "picture" and obtain

$$
H^{1 I}=\frac{\Omega}{\sqrt{6}}\left(\left|\phi_{14}\right\rangle-\left|\phi_{1}\right\rangle\right)\left\langle\varphi_{2}\left|+\frac{\Omega}{\sqrt{6}}\right| \varphi_{2}\right\rangle\left(\left\langle\phi_{14}|-|\left\langle\phi_{1}\right|\right)+\Delta\left|\varphi_{2}\right\rangle\left\langle\varphi_{2}\right| .\right.
$$

One can find that the terms including $\left|\varphi_{3}\right\rangle\left\langle\varphi_{3}|,| \varphi_{4}\right\rangle\left\langle\varphi_{4}|,| \varphi_{5}\right\rangle\left\langle\varphi_{5}|,| \varphi_{6}\right\rangle\left\langle\varphi_{6}|,| \varphi_{7}\right\rangle\left\langle\varphi_{7}\right|$, $\left|\varphi_{8}\right\rangle\left\langle\varphi_{8}|,| \varphi_{9}\right\rangle\left\langle\varphi_{9}|,| \varphi_{10}\right\rangle\left\langle\varphi_{10}|,| \varphi_{11}\right\rangle\left\langle\varphi_{11}\right|$, and $\left|\varphi_{12}\right\rangle\left\langle\varphi_{12}\right|$ are discarded since they are decoupled to the encoded qubit. If we consider $|\Psi\rangle=\frac{1}{\sqrt{2}}\left(\left|\phi_{14}\right\rangle-\left|\phi_{1}\right\rangle\right)$ as a stable level, we can regard Eq. (17) as an effective Hamiltonian of the two-level system. Thus the stable level is coupled to the excited level $\left|\varphi_{2}\right\rangle$ with coupling constant $\frac{\Omega}{\sqrt{3}}$ and detuning $\Delta$. On the large detuning condition $(\Delta \gg \Omega)$, there is no transition between $|\Psi\rangle$ and $\left|\varphi_{2}\right\rangle$, only the stark shift contributes to the variation of energy for each level [27]. By adiabatically eliminating the excited state $\left|\varphi_{2}\right\rangle$, we obtain the final effective Hamiltonian

$$
H_{e f f}^{1 I}=-\frac{\Omega^{2}}{6 \Delta}\left(\left|\phi_{1}\right\rangle\left\langle\phi_{1}|+| \phi_{14}\right\rangle\left\langle\phi_{14}\right|\right)+\frac{\Omega^{2}}{6 \Delta}\left(\left|\phi_{1}\right\rangle\left\langle\phi_{14}|+| \phi_{1}\right\rangle\left\langle\phi_{14}\right|\right) .
$$

For an interaction time $t$, the state of the whole system becomes

$$
|\Phi(t)\rangle=\frac{1}{2}\left(1+\cos \frac{\Omega^{2} t}{3 \Delta}+i \sin \frac{\Omega^{2} t}{3 \Delta}\right)\left|\phi_{1}\right\rangle+\frac{1}{2}\left(1-\cos \frac{\Omega^{2} t}{3 \Delta}-i \sin \frac{\Omega^{2} t}{3 \Delta}\right)\left|\phi_{14}\right\rangle .
$$

If we choose $\frac{\Omega^{2} t}{3 \Delta}=\pi$ and the final state becomes $\left|\Phi\left(\frac{3 \Delta \pi}{\Omega^{2}}\right)\right\rangle=\left|\phi_{14}\right\rangle$, one will obtain the transform: $\left|\phi_{1}\right\rangle=\left|g_{0}\right\rangle_{1}\left|g_{L}\right\rangle_{2}\left|g_{R}\right\rangle_{3}|00\rangle \rightarrow\left|\phi_{14}\right\rangle=\left|g_{0}\right\rangle_{1}\left|g_{R}\right\rangle_{2}\left|g_{L}\right\rangle_{3}|00\rangle$.

If the initial state is $\left|g_{0}\right\rangle_{1}\left|g_{R}\right\rangle_{2}\left|g_{L}\right\rangle_{3}|00\rangle$, a analogue method is utilized with the initial state $\left|g_{0}\right\rangle_{1}\left|g_{L}\right\rangle_{2}\left|g_{R}\right\rangle_{3}|00\rangle$. As a result, the interaction time is also $t=\frac{3 \Delta \pi}{\Omega^{2}}$ when the final state becomes $\left|g_{0}\right\rangle_{1}\left|g_{L}\right\rangle_{2}\left|g_{R}\right\rangle_{3}|00\rangle$.

If the initial state is $\left|g_{0}\right\rangle_{1}\left|g_{L}\right\rangle_{2}\left|g_{L}\right\rangle_{3}|00\rangle$, the whole system evolves in a closed subspace spanned by $\left\{\left|\phi_{1}^{\prime}\right\rangle,\left|\phi_{2}^{\prime}\right\rangle,\left|\phi_{3}^{\prime}\right\rangle,\left|\phi_{4}^{\prime}\right\rangle,\left|\phi_{5}^{\prime}\right\rangle,\left|\phi_{6}^{\prime}\right\rangle,\left|\phi_{7}^{\prime}\right\rangle,\left|\phi_{8}^{\prime}\right\rangle\right\}$. Then we can rewrite the above Hamil- 
tonian in the " $H_{c a}^{2}$ " representation:

$$
\begin{aligned}
H_{t o t}^{2}= & H_{c a}^{2}+H_{l a}^{2}+H_{d e}^{2}, \\
H_{c a}^{2}= & -g\left|\varphi_{2}^{\prime}\right\rangle\left\langle\varphi_{2}^{\prime}|-g| \varphi_{3}^{\prime}\right\rangle\left\langle\varphi_{3}^{\prime}|+g| \varphi_{4}^{\prime}\right\rangle\left\langle\varphi_{4}^{\prime}|+g| \varphi_{5}^{\prime}\right\rangle\left\langle\varphi_{5}^{\prime}|-2 g| \varphi_{6}^{\prime}\right\rangle\left\langle\varphi_{6}^{\prime}|+2 g| \varphi_{7}^{\prime}\right\rangle\left\langle\varphi_{7}^{\prime}\right|, \\
H_{l a}^{2}= & \frac{\Omega}{2 \sqrt{3}}\left(-\left|\varphi_{2}^{\prime}\right\rangle+\left|\varphi_{4}^{\prime}\right\rangle\right)\left\langle\phi _ { 1 } ^ { \prime } \left|+\frac{\Omega}{2}\left(-\left|\varphi_{3}^{\prime}\right\rangle+\left|\varphi_{5}^{\prime}\right\rangle\right)\left\langle\phi _ { 1 } ^ { \prime } \left|+\frac{\Omega}{2 \sqrt{6}}\left(-\left|\varphi_{6}^{\prime}\right\rangle+\left|\varphi_{7}^{\prime}\right\rangle\right)\left\langle\phi_{1}^{\prime}\right|,\right.\right.\right.\right. \\
H_{d e}^{2}= & \frac{\Delta}{2}\left(\left|\varphi_{2}^{\prime}\right\rangle-\left|\varphi_{4}^{\prime}\right\rangle\right)\left(\left\langle\varphi_{2}^{\prime}\left|-\left\langle\varphi_{4}^{\prime}\right|\right)+\frac{\Delta}{2}\left(\left|\varphi_{3}^{\prime}\right\rangle-\left|\varphi_{5}^{\prime}\right\rangle\right)\left(\left\langle\varphi_{3}^{\prime}\right|-\left\langle\varphi_{5}^{\prime}\right|\right)\right.\right. \\
& +\frac{\Delta}{2}\left(\left|\varphi_{6}^{\prime}\right\rangle-\left|\varphi_{7}^{\prime}\right\rangle\right)\left(\left\langle\varphi_{6}^{\prime}\right|-\left\langle\varphi_{7}^{\prime}\right|\right) .
\end{aligned}
$$

A analogue method is utilized with the Eq. (16) - Eq. (18). We can obtain that the final effective Hamiltonian $H_{e f f}^{2 I}$ has no effect on the time evolution of initial state $\left|g_{0}\right\rangle_{1}\left|g_{L}\right\rangle_{2}\left|g_{L}\right\rangle_{3}|00\rangle$. Namely,

$$
H_{e f f}^{2 I}\left|g_{0}\right\rangle_{1}\left|g_{L}\right\rangle_{2}\left|g_{L}\right\rangle_{3}|00\rangle=0
$$

Therefore, the initial state $\left|g_{0}\right\rangle_{1}\left|g_{L}\right\rangle_{2}\left|g_{L}\right\rangle_{3}|00\rangle$ remains unchanged during the time evolution.

Specially, it is a similar case to the initial state $\left|g_{0}\right\rangle_{1}\left|g_{R}\right\rangle_{2}\left|g_{R}\right\rangle_{3}|00\rangle$. The final state does not have any change during the evolution. As a result, we can also acquire the Fredkin gate in the detuning model.

\section{NUMERICAL ANALYSIS AND DISCUSSIONS}

All the above derivations are based on the ideal case that the influence of decoherence induced by cavity decay and atomic spontaneous emission on the time evolution of system is omitted. We here utilize a quantum jump approach [28-30] to discuss the influence of decoherence on the time evolution of system. If no photon is detected through the leakage from the cavity and the atomic spontaneous emission, the time evolution of system is dominated by the conditional Hamiltonian

$$
H_{\text {cond }}=H_{\text {total }}-\sum_{k=1}^{3} \sum_{j=L, R}\left(i \frac{\gamma_{j}}{2}\left|e_{0}\right\rangle_{k}\left\langle e_{0}\right|+i \frac{\kappa_{j}}{2} a_{j}^{\dagger} a_{j}\right)
$$

where $\gamma_{j}$ is spontaneous emission rate for the excited state $\left|e_{0}\right\rangle$ and $\kappa_{j}$ is decay rate of the corresponding cavity mode. Without loss of generality, we set $\gamma_{j}=\gamma$ and $\kappa_{j}=\kappa$. Assuming the initially state $|\Phi(0)\rangle=\frac{1}{2 \sqrt{2}}\left(g_{0}+g_{R}\right) \otimes\left(g_{L}+g_{R}\right) \otimes\left(g_{L}+g_{R}\right)$, the time evolution of system 


$$
|\Phi(t)\rangle=\frac{U_{\text {cond }}(t)|\Phi(0)\rangle}{\sqrt{P_{\text {suc }}(t)}},
$$

where $U_{\text {cond }}(t)=e^{-i H_{\text {cond }} t}$ is the time evolution operator for $H_{\text {cond }}, P_{\text {suc }}(t)=$ $\left\langle\Phi(0)\left|U_{\text {cond }}^{\dagger}(t) U_{\text {cond }}(t)\right| \Phi(0)\right\rangle$ represents that no photon has been emitted at time $t$. The computation will fail and has to be repeated if photons are emitted. To some extent, it can be made up by monitoring photon emissions with good detectors.

Fig. 3 (Fig. 4) shows the relation among the fidelity $F$ (the success probability $P$ ) of the Fredkin gate and cavity decay and atomic spontaneous emission with the other parameter chosen as $\Omega=0.03 \mathrm{~g}$ in the resonant model. One can see from Fig. 3 that with the increasing of cavity decay and atomic spontaneous emission, the fidelity $F$ of the Fredkin gate will decrease. If we set $\gamma / g=0.1$ and $\kappa / g=0.1$, one can obtain the value of fidelity $F=77.78 \%$ and success probability $P=88.66 \%$. However, we can see from Fig. 3 that this model is robust against cavity decay due to the fidelity $F$ is about $99.75 \%$ even though cavity decay $\kappa / g=0.1$ when spontaneous emission $\gamma / g=0$. That is due to the fact that the system evolves in a closed subspace where the cavity mode field is not excitation. Thus the main decoherence of the resonant model is spontaneous emission because the evolution of whole system involves excited states of the atoms. As a result, the resonant model is suitable for small atomic spontaneous emission rate and large cavity decay rate.

Fig. 5 (Fig. 6) shows the relation among the fidelity $F$ (the success probability $P$ ) of the Fredkin gate and cavity decay and atomic spontaneous emission with the other parameters chosen as $\Omega=0.03 g$ and $\Delta=0.3 g$ in the large detuning model. One can see from Fig. 5 (Fig. 6) that with the increasing of cavity decay and atomic spontaneous emission, the fidelity $F$ (the success probability $P$ ) of the Fredkin gate will decrease. If we set $\gamma / g=0.1$ and $\kappa / g=0.1\left(g^{2}=100 \kappa \gamma\right)$, one can obtain the value of fidelity $F=97.83 \%$ and success probability $P=84.60 \%$. As a result, the fidelity $F$ is insensitive to cavity decay. That is also due to the fact that the system evolves in a closed subspace where the cavity mode field is not excitation. On the other hand, the fidelity $F$ is also insensitive to atomic spontaneous emission because of the presence of the large detuning, eliminating the excited state of atoms adiabatically. As a result, the lage detuning model is suitable for moderate values of atomic spontaneous emission rate and cavity decay rate and the lifetime of the states should be long enough because the interaction time needs longer compared to the resonant model. Thus 
high fidelity of the proposed Fredkin gate can be obtained for the optimal value of the large detuning and also depends on the practical experimental parameters restriction of cavity decay and atomic spontaneous emission.

\section{EXPERIMENTAL FEASIBILITY AND CONCLUSIONS}

Finally, we give a brief discussion on the experimental feasibility of both models. The atomic configuration involved in our models can be implemented with ${ }^{87} \mathrm{Rb}$. The relevant atomic levels are shown in Fig. 7. Each atom is assumed to be coupled to $\sigma^{+}$and $\sigma^{-}$ -polarized photon modes of the bi-modal cavity and the first atom is coupled to an external $\pi$-polarized classical field individually. In our models, all the encoded qubit states are lowenergy states and the cavity modes are almost in the vacuum state during the time evolution. On the other hand, it is feasible with the parameters $g=2 \pi \times 750 \mathrm{MHz}, \gamma=2 \pi \times 2.62$ $\mathrm{MHz}, \kappa=2 \pi \times 3.5 \mathrm{MHz}$ in an optical cavity with the wavelength in the region $630-850$ $\mathrm{nm}$ in recent experiments [31, 32]. In the resonant model, the fidelity will be about $99.73 \%$ and the interaction time $t \simeq 0.0385 \mu$ s. We will obtain a high fidelity about $99.93 \%$ and the interaction time $t \simeq 0.667 \mu \mathrm{s}$ in the detuning model. Therefore, it allows construction of an atomic system for quantum computation in the presence of decoherence.

In summary, we have studied one-step implementation of the Fredkin gate in a bi-modal cavity under both resonant and large detuning conditions based on quantum Zeno dynamics, which reduces the complexity of experiment operations. The principle implementing the Fredkin gate is quite different in both models which each of them has its respective advantages. Therefore, it provides a flexibility to adapt the proposed model to different experimental apparatus. In the resonant model, it is robust against the cavity decay because the state keeps in a subspace without exciting the cavity field during the evolution and the interaction time needs rather short because of the resonant interaction. However atomic spontaneous emission is the main decoherence in this model. In the large detuning model, it is insensitive to the influence of decoherence caused by decay of the cavity modes and spontaneous of the excited states due to the large detuning condition. However the time is much longer than the time required in the resonant model on the same Rabi frequency $\Omega$ and the same coupling strength $g$. In addition, we have also briefly discussed the influence of decoherence induced by cavity decay and atomic spontaneous emission by numerical 
calculation. Therefore, we hope that with the current experimental technology it may be possible to implement the Fredkin gate in this paper.

\section{Acknowledgments}

This work was supported by the Natural Science Foundation of Fuzhou University of China under grant no 022264 and no 2010-XQ-28, the funds from Education Department of Fujian Province of China under grant no JB08010, no JA10009 and no JA10039, the National Natural Science Foundation of Fujian Province of China under grant No. 2009J06002 and no 2010J01006, the National Natural Science Foundation of China under grant no 11047122, no 10875020 and 10974028, Doctoral Foundation of the Ministry of Education of China under grant no 20093514110009, and China Postdoctoral Science Foundation under grant no 20100471450 .

[1] L. K. Grover (1998), Quantum computers can search rapidly by using almost any transformation, Phys. Rev. Lett. 80, pp. 4329-4332.

[2] S. B. Zheng and G. C. Guo (2000), Efficient scheme for two-atom entanglement and quantum information processing in cavity QED, Phys. Rev. Lett. 85, pp. 2392-2395.

[3] C. P. Yang, S. Chu, and S. Han (2004), Simplified realization of two-qubit quantum phase gate with four-level systems in cavity QED, Phys. Rev. A 70, 044303.

[4] S. B. Zheng (2005), Quantum logic gates for two atoms with a single resonant interaction, Phys. Rev. A 71, 062335.

[5] Z. B. Yang, H. Z. Wu, W. J. Su, and S. B. Zheng (2009), Quantum phase gates for two atoms trapped in separate cavities within the null- and single-excitation subspaces, Phys. Rev. A 80, 012305 .

[6] T. Monz, K. Kim, W. Hänsel, M. Riebe, A. S. Villar, P. Schindler, M. Chwalla, M. Hennrich, and R. Blatt (2009), Realization of the quantum Toffoli gate with trapped ions, Phys. Rev. Lett. 102, 040501.

[7] G. J. Milburn (1989), Quantum optical Fredkin gate, Phys. Rev. Lett. 62, pp. 2124-2127.

[8] J. Fiurášek (2006), Linear-optics quantum Toffoli and Fredkin gates, Phys. Rev. A 73, 062313. 
[9] T. C. Ralph, K. J. Resch, and A. Gilchrist (2007), Efficient Toffoli gates using qudits, Phys. Rev. A 75, 022313.

[10] X. Q. Shao, A. D. Zhu, S. Zhang, J. S. Chung, and K. H. Yeon (2007), Efficient scheme for implementing an $N$-qubit Toffoli gate by a single resonant interaction with cavity quantum electrodynamics, Phys. Rev. A 75, 034307.

[11] B. P. Lanyon, M. Barbieri, M. P. Almeida, T. Jennewein, T. C. Ralph, K. J. Resch, G. J. Pryde, J. L. OBrien, A. Gilchrist, and A. G. White (2008), Quantum computing using shortcuts through higher dimensions, e-print arXiv:0804.0272.

[12] Y. X. Gong, G. C. Guo, and T. C. Ralph (2008), Methods for a linear optical quantum Fredkin gate, Phys. Rev. A 78, 012305.

[13] J. Fiurášek (2008), Linear optical Fredkin gate based on partial-SWAP gate, Phys. Rev. A 78, 032317.

[14] X. Q. Shao, L. Chen, S. Zhang, and Y. F. Zhao (2009), Swap gate and controlled swap gate based on a single resonant interaction with cavity quantum electrodynamics, Phys. Scr. 79, 065004 .

[15] B. Misra and E. C. G. Sudarshan (1977), The Zeno's paradox in quantum theory, J. Math. Phys. 18, pp. 756-763.

[16] P. Facchi, V. Gorini, G. Marmo, S. Pascazio, and E. C. G. Sudarshan (2000), Quantum Zeno dynamics, Phys. Lett. A 275, pp. 12-19.

[17] P. Facchi, S. Pascazio, A. Scardicchio, and L. S. Schulman (2001), Zeno dynamics yields ordinary constraints, Phys. Rev. A 65, 012108.

[18] P. Facchi and S. Pascazio (2001), Quantum Zeno and inverse quantum Zeno effects, Progress in Optics, 42, pp. 147-217.

[19] A. Beige, D. Braun, B. Tregenna, and P. L. Knight (2000), Quantum computing using dissipation to remain in a decoherence-free subspace, Phys. Rev. Lett. 85, pp. 1762-1765.

[20] J. Pachos and H. Walther (2002), Quantum computation with trapped ions in an optical cavity, Phys. Rev. Lett. 89, 187903.

[21] J. K. Pachos, and A. Beige (2004), Decoherence-free dynamical and geometrical entangling phase gates, Phys. Rev. A 69, 033817.

[22] X. Q. Shao, H. F. Wang, L. Chen, S. Zhang, and K. H. Yeon (2009), One-step implementation of the Toffoli gate via quantum Zeno dynamics, Phys. Lett. A 374, pp. 28-33. 


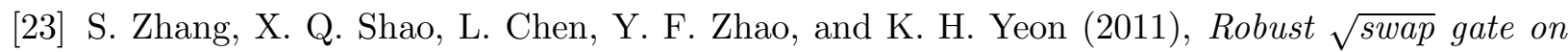
nitrogen-vacancy centres via quantum Zeno dynamics, J. Phys. B: At. Mol. Opt. Phys. 44, 075505 .

[24] Z. C. Shi, Y. Xia, J. Song, and H. S. Song (2011), Atomic quantum state transferring and swapping via quantum Zeno dynamics, J. Opt. Soc. Am. B 28, pp. 2909-2914.

[25] P. Facchi and S. Pascazio (2002), Quantum Zeno subspaces, Phys. Rev. Lett. 89, 080401.

[26] P. Facchi, G. Marmo, and S. Pascazio (2009), Quantum Zeno dynamics and quantum Zeno subspaces, J. Phys: Conf. Ser. 196, 012017.

[27] D. F. James and J. Jerke (2007), Effective Hamiltonian theory and its applications in quantum information, Can. J. Phys. 85, pp. 625-632.

[28] M. B. Plenio and P. L. Knight (1998), The quantum-jump approach to dissipative dynamics in quantum optics, Rev. Mod. Phys. 70, pp. 101-144.

[29] G. Hegerfeldt and D. Sondermann (1996), Conditional Hamiltonian and reset operator in the quantum jump approach, Quantum Semiclass. Opt. 8, pp. 121-132.

[30] J. Dalibard, Y. Castin, and K. Mølmer (1992), Wave-function approach to dissipative processes in quantum optics, Phys. Rev. Lett. 68, pp. 580-583.

[31] S. M. Spillane, T. J. Kippenberg, K. J. Vahala, K. W. Goh, E. Wilcut, and H. J. Kimble (2005), Ultrahigh-Q toroidal microresonators for cavity quantum electrodynamics, Phys. Rev. A 71, 013817.

[32] J. R. Buck and H. J. Kimble (2003), Optimal sizes of dielectric microspheres for cavity QED with strong coupling, Phys. Rev. A 67, 033806. 
FIG. 1. The level configuration of the atoms (the resonant model).

FIG. 2. The level configuration of the atoms (the large detuning model).

FIG. 3. The influence of cavity decay and atomic spontaneous emission on the fidelity of the Fredkin gate (the resonant model). The other parameter is $\Omega=0.03 \mathrm{~g}$.

FIG. 4. The influence of cavity decay and atomic spontaneous emission on the success probability of the Fredkin gate (the resonant model). The other parameter is $\Omega=0.03 g$.

FIG. 5. The influence of cavity decay and atomic spontaneous emission on the fidelity of the Fredkin gate (the large detuning model). The other parameters are $\Omega=0.03 g, \Delta=0.3 g$.

FIG. 6. The influence of cavity decay and atomic spontaneous emission on the success probability of the Fredkin gate (the large detuning model). The other parameters are $\Omega=$ $0.03 g, \Delta=0.3 g$.

FIG. 7. The energy levels of ${ }^{87} \mathrm{Rb}$ (left side denotes the resonant model and right side denotes the large detuning model). 

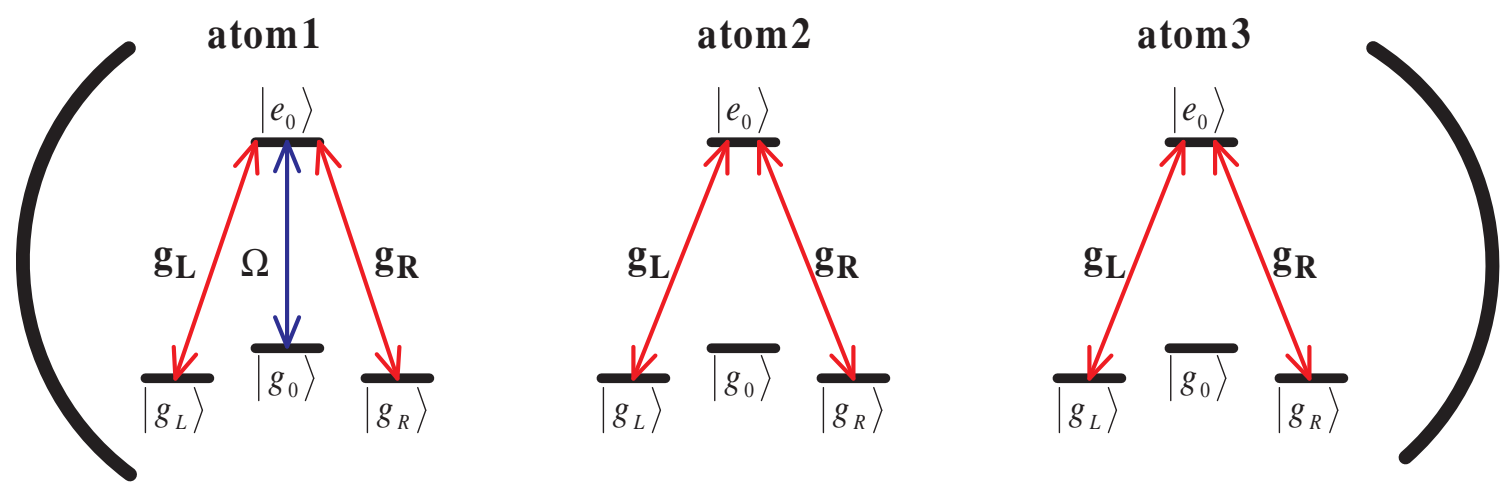

FIG. 1:
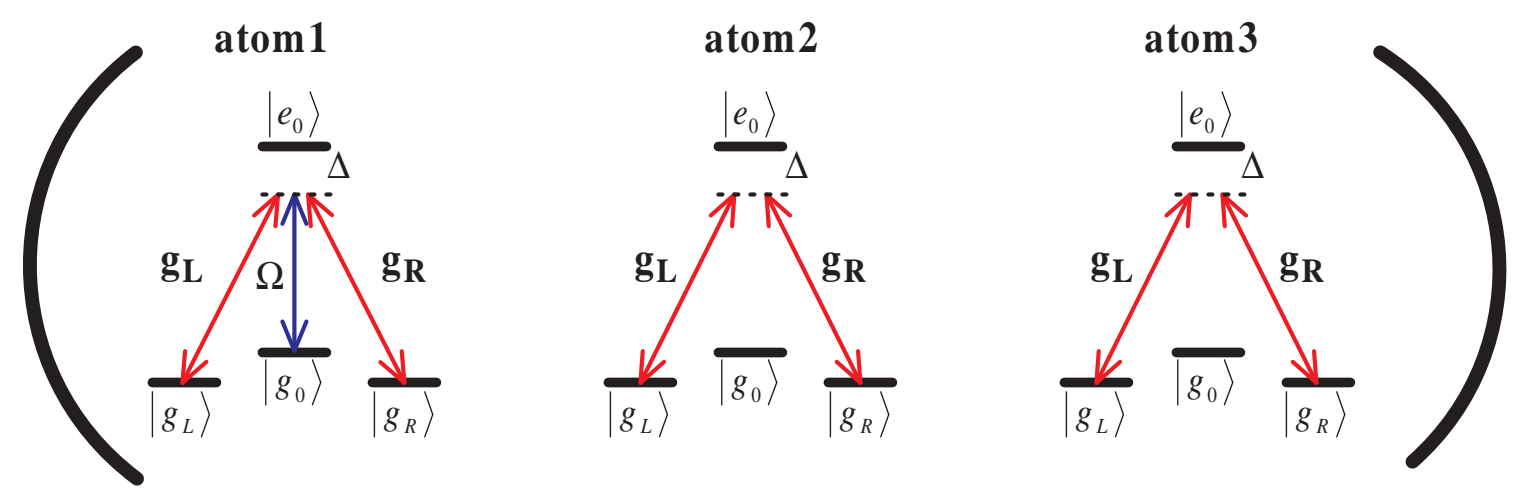

FIG. 2: 


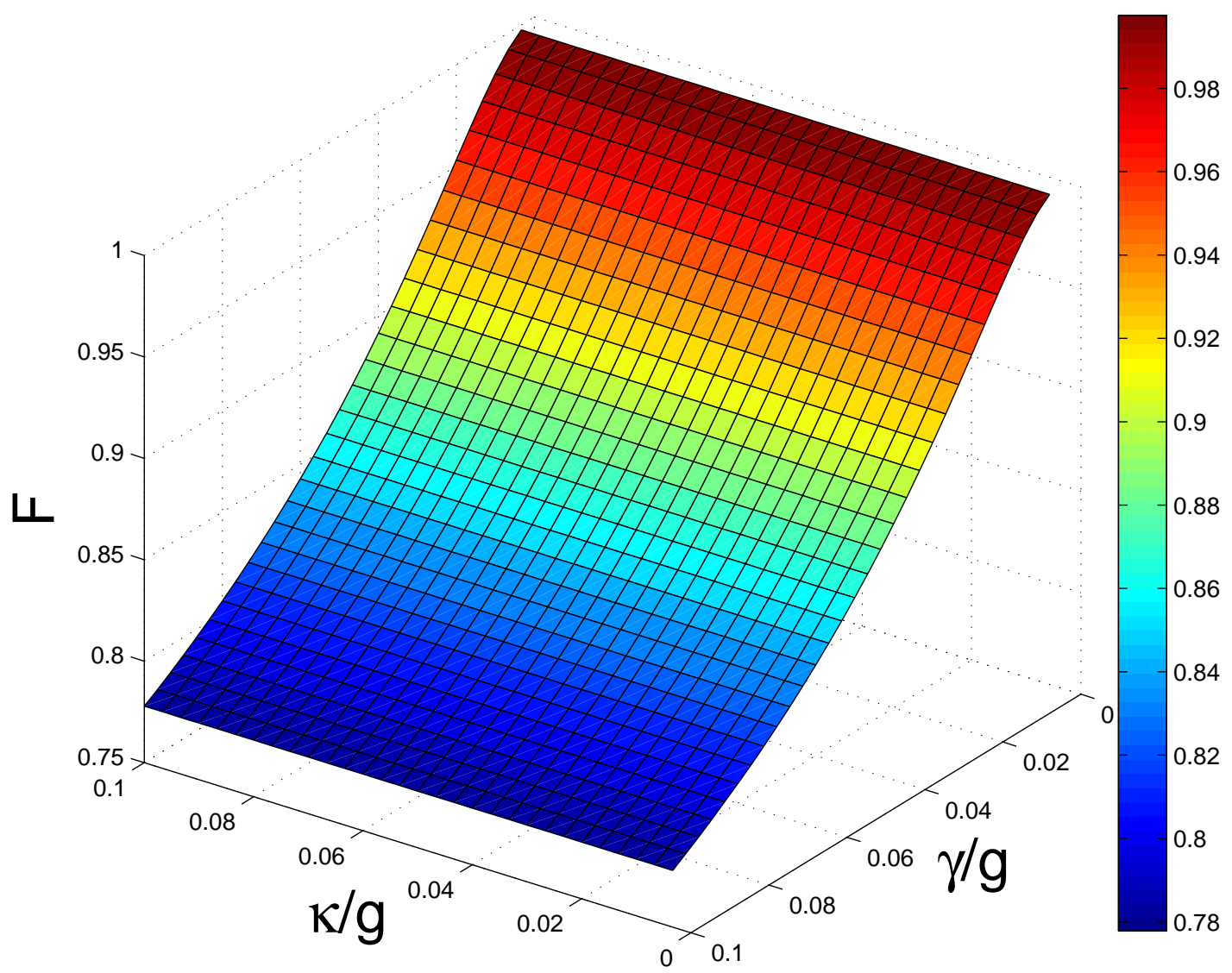

FIG. 3: 


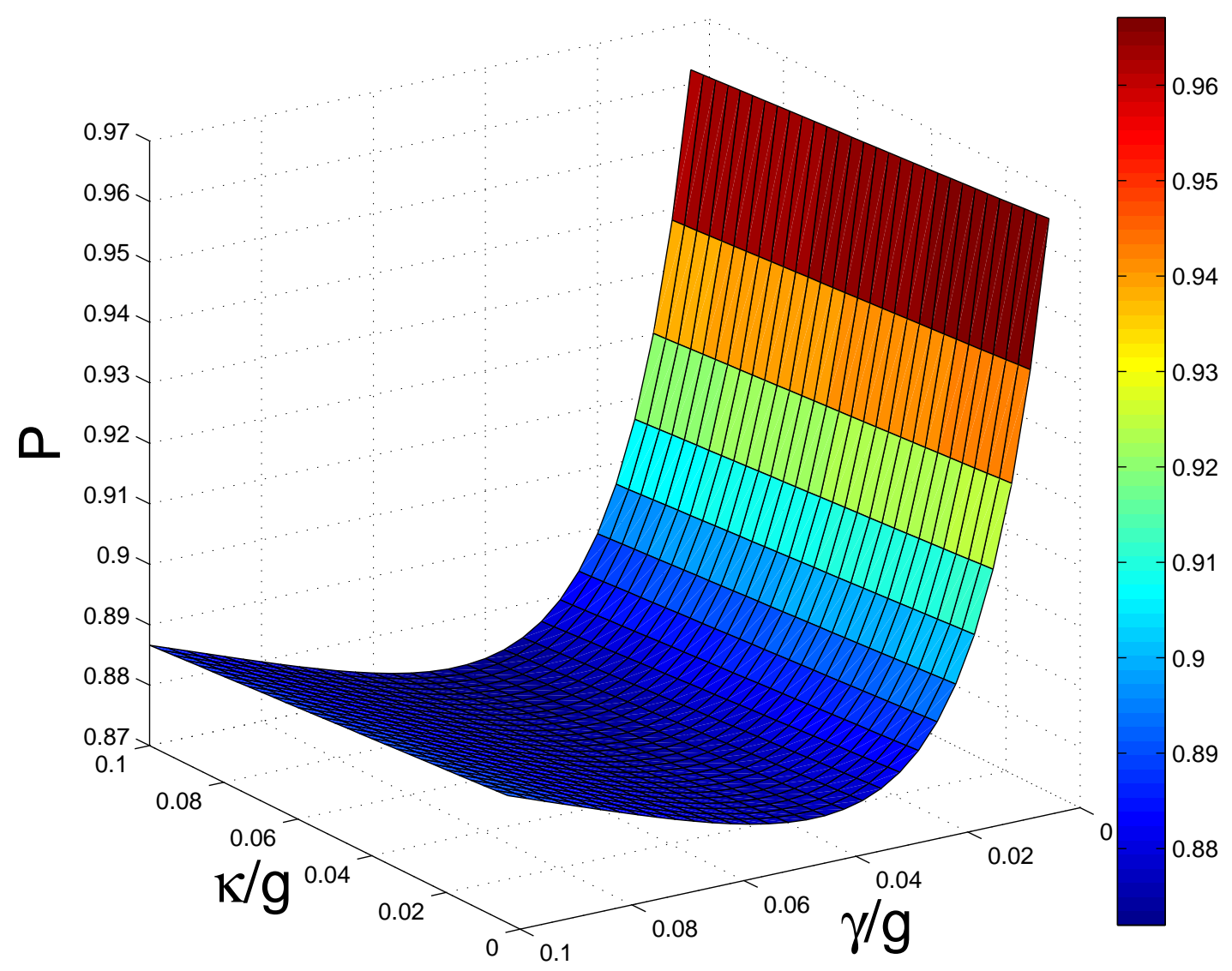

FIG. 4: 


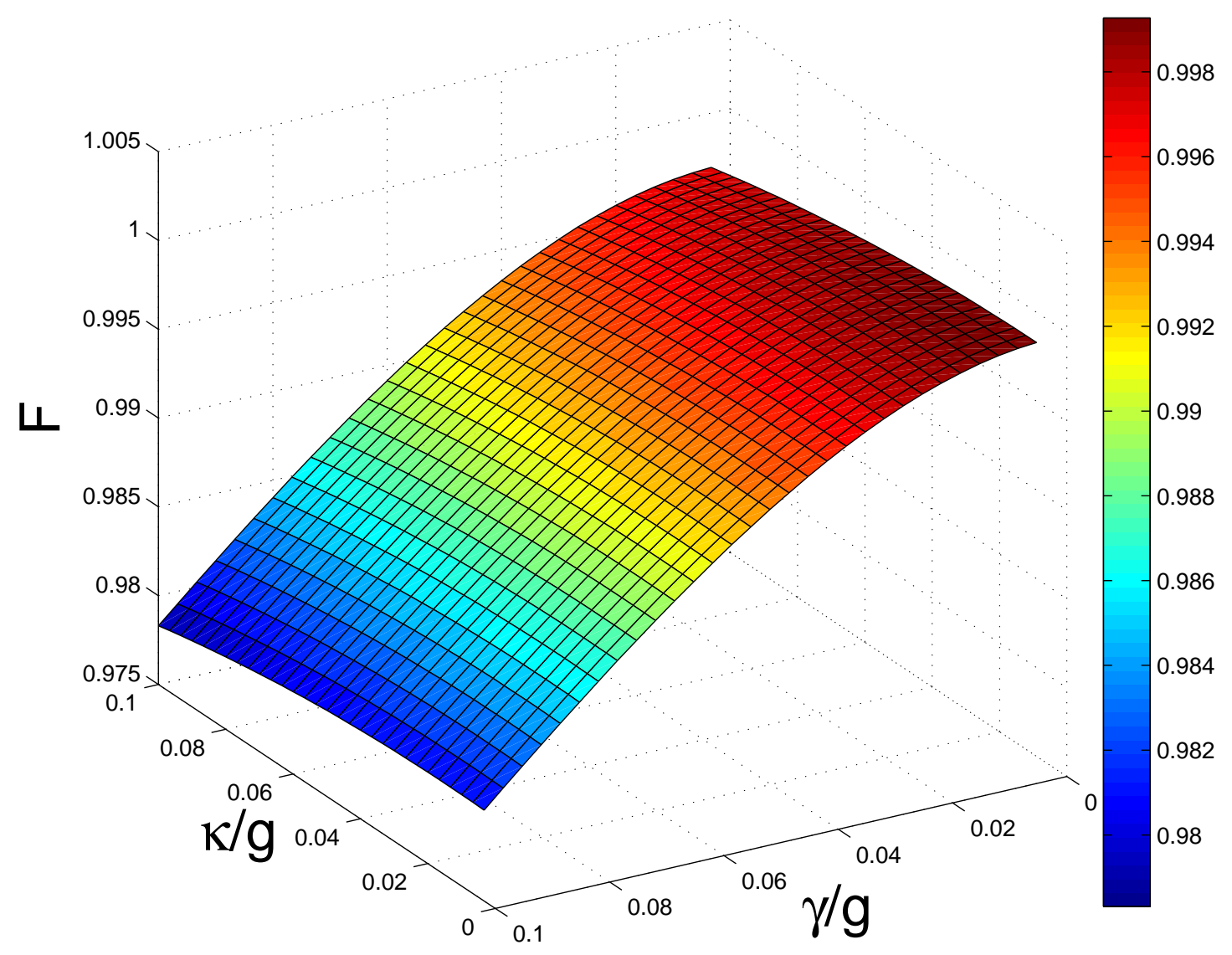

FIG. 5: 


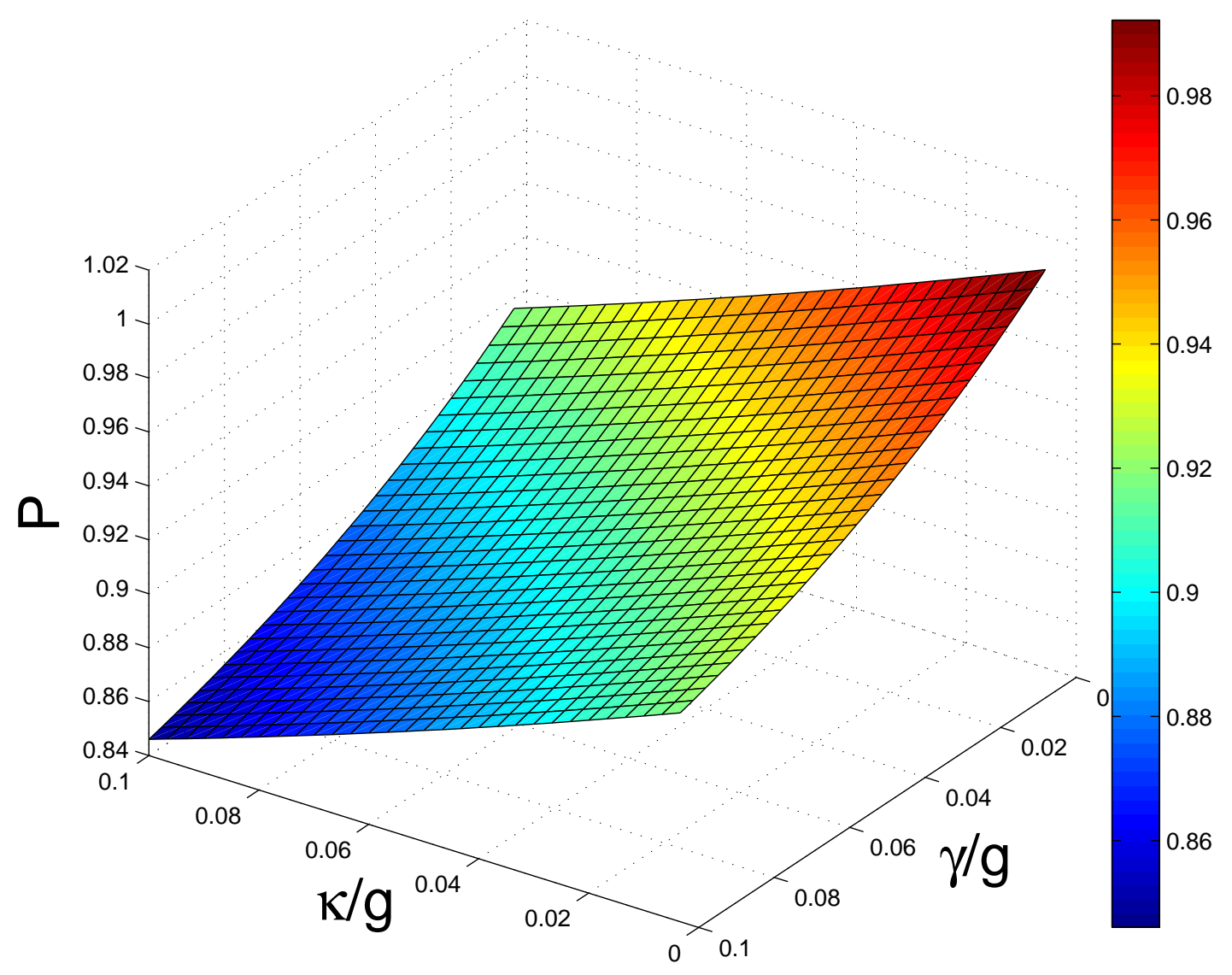

FIG. 6:
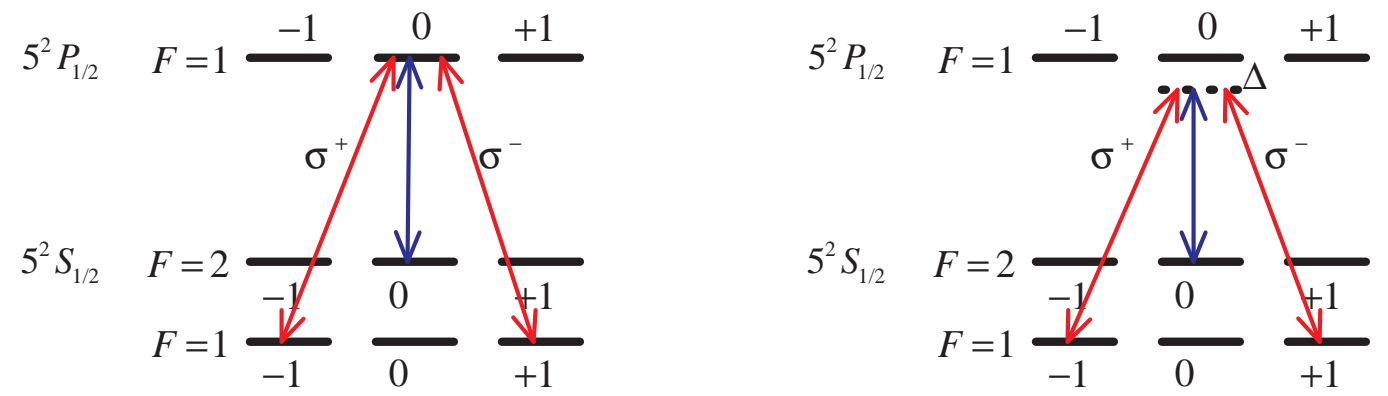

FIG. 7: 